\title{
The D0 Silicon Tracker
}

\author{
W. E. Cooper, for the D0 Collaboration* \\ Fermi National Accelerator Laboratory, Batavia, Illinois 60510-5011, United States of America
}

Elsevier use only: Received date here; revised date here; accepted date here

\begin{abstract}
The D0 silicon micro-strip tracker, which was installed for Run II of the Fermilab Collider, and an inner silicon micro-strip layer 0, which was installed for Run IIb of the Collider, are described.
\end{abstract}

(C) 2008 Elsevier B.V. All rights reserved

29.40.Gx; 29.40.Wk

Silicon; micro-strip; tracker; detector

\section{Introduction}

The D0 Silicon Tracker comprises two portions: an original set of barrels and disks built for Run IIa of the Tevatron Collider [1][2] and an additional "Layer 0", built for Run IIb of the Collider. The original portions of the tracker have been in operation since spring 2001. To address concerns regarding possible deterioration of the inner layers of that detector due to radiation damage and to improve vertex resolution, an inner Layer 0 was added for Run IIb of the Collider. Layer 0 has been in operation since spring 2006.

\section{Run IIa silicon}

The 792576 channel, 1248 sensor, Run IIa silicon tracker includes a central portion with six barrels, each with four layers, six "F-disks", one attached to each barrel, and six more F-disks divided evenly between two end disk modules. The general arrangement of Run IIa silicon within surrounding detector elements is shown in figure 1. The central silicon elements are supported from the inner surface of central scintillating fiber tracker (CFT) barrel 1 via two double-walled cylinders made of carbon fiber - epoxy laminate. Barrel sensor centerline radii range from 27.1 to $100.6 \mathrm{~mm}$. F-disks cover a similar radial range. The barrels and F-disks of the central silicon occupy a $1066 \mathrm{~mm}$ long region and are arrayed

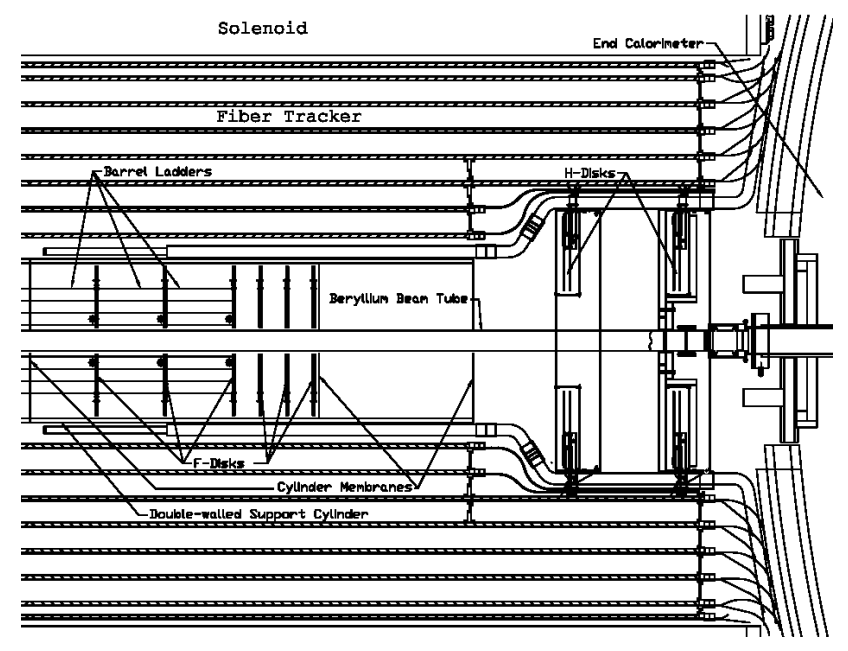

Fig. 1. Plan view of the Run IIa silicon tracker within surrounding elements of the D0 detector.

symmetrically about the center of the interaction diamond. For tracks from the origin and at the least favorable azimuth, the central silicon provides three or more hits to $\eta= \pm 3.54$.

Four "H-disks", with silicon centered at $\mathrm{z}= \pm 1004 \mathrm{~mm}$ and $\mathrm{z}= \pm 1210 \mathrm{~mm}$, are supported independently from the inner surface of scintillating fiber tracker barrel 3. The H-disks, whose silicon extends from $\mathrm{R}=96 \mathrm{~mm}$ to $\mathrm{R}=236 \mathrm{~mm}$,

* Corresponding author. Tel.: +1-630-840-4093; fax: +1-630-840-8886.

E-mail address: cooper@fnal.gov. 
complete the tracker and allow more precise momentum measurements of forward tracks.

Central silicon is supported from two half-length, doublewalled carbon fiber laminate cylinders with removable access covers. Signals from individual ladders and wedges are brought on hybrid "pigtails" to the outer surface of the support cylinder. There they are connected to low mass cables which run longitudinally.

All D0 silicon is cooled by a $32 \%$ by volume mixture of ethylene glycol in water. Coolant is drawn from an atmospheric pressure reservoir, which ensures that coolant pressure within the detector region is sub-atmospheric. No leaks, blockages, corrosion, or other problems have been observed in the detector region since the silicon was commissioned. Typical coolant temperature is $-8.5^{\circ} \mathrm{C}$, which leads to a maximum temperature of $+2^{\circ} \mathrm{C}$ at any point within a Run IIa sensor. To ensure that condensation of moisture will not occur, the silicon region is purged with dry air having a dew-point which is typically below $-50^{\circ} \mathrm{C}$.

\subsection{Sensors}

Seven varieties of sensors were used in the Run IIa silicon tracker. Please see table 1. All sensors are AC coupled and nominally $300 \mu \mathrm{m}$ thick. All barrel and F-disk sensors were obtained from Micron Semiconductor, Ltd., Eurisys, or CSEM. All H-disk sensors were obtained from ELMA.

Table 1. Sensor types

\begin{tabular}{|l|l|l|}
\hline Location & Type & Pitch $(\mu \mathrm{m})$ \\
\hline $\begin{array}{l}\text { Central } 4 \text { barrels, layers } \\
1 \text { and 3 }\end{array}$ & $\begin{array}{l}\text { Double-sided, double- } \\
\text { metal, } 90^{\circ} \text { stereo }\end{array}$ & $\begin{array}{l}50 \text {, axial surface } \\
153.5, \text { stereo surface }\end{array}$ \\
\hline $\begin{array}{l}\text { Outer } 2 \text { barrels, layers } \\
1 \text { and 3 }\end{array}$ & Single-sided, axial & 50 \\
\hline $\begin{array}{l}\text { All barrels, layers 2 and } \\
4\end{array}$ & Double-sided, $2^{\circ}$ stereo & $\begin{array}{l}50, \text { axial surface } \\
62.5, \text { stereo surface }\end{array}$ \\
\hline F-disks & $\begin{array}{l}\text { Double-sided, 30 } \\
\text { included stereo angle }\end{array}$ & $\begin{array}{l}50, \text { p-side } \\
62.5, \text { n-side }\end{array}$ \\
\hline H-disks & $\begin{array}{l}\text { Single-sided, mated } \\
\text { back to back to provide } \\
15^{\circ} \text { stereo }\end{array}$ & $\begin{array}{l}40, \text { trace pitch } \\
80, \text { readout pitch }\end{array}$ \\
\hline
\end{tabular}

\subsection{Sensor support, cooling, and readout connections}

Sensors of barrels are formed into ladders of nominal length $120 \mathrm{~mm}$. The 72 ladders of each barrel are supported from a $9.525 \mathrm{~mm}$ thick "cooled" beryllium bulkhead, which connects to the silicon support cylinder via leaf springs and adjustable mounts at 3, 6, and 9 o'clock. A $0.762 \mathrm{~mm}$ thick "passive" beryllium bulkhead locates the far ends of ladders relative to one another. Coolant flows through two passages of typical flow cross-section $5.842 \mathrm{~mm} \times 7.366 \mathrm{~mm}$ within each cooled bulkhead. Please see figure 2 .

SVX-2 readout chips on multi-layer "high density interconnects" (HDI's) are mounted via beryllium substrates

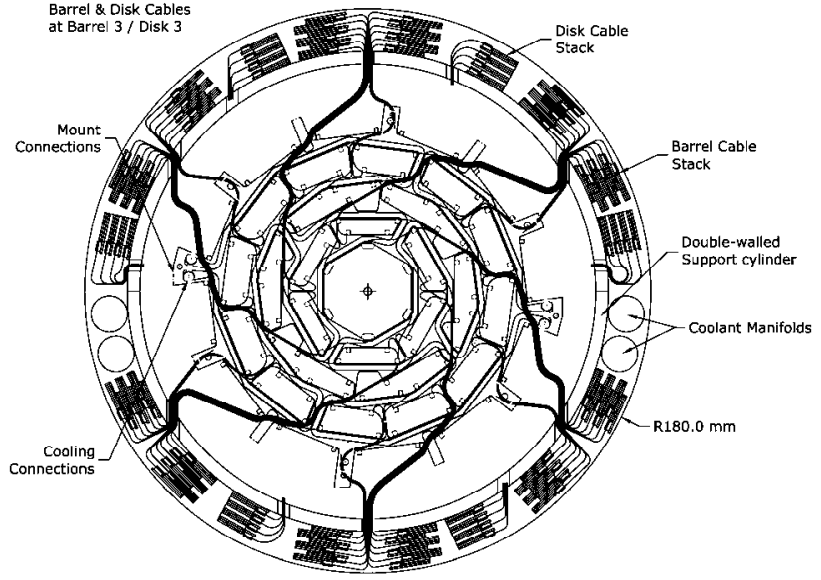

Fig. 2. End view of ladder positions on the cooled bulkhead of barrel 3. The two coolant passages per bulkhead, three locations for connections to kinematic mounts, protrusions for disk support posts, support cylinder, and low-mass cable bundles and coolant distribution manifolds on the exterior of the support cylinder are shown.

to the silicon surface of each ladder. "Pigtail" extensions of the HDI's carry signals to and from the outer periphery of the silicon support cylinder. On the outer periphery, each pigtail is connected to a low-mass cable running longitudinally along the support cylinder. Hirose, zero insertion force connectors are used for the connections to low-mass cables.

Sensors of F-disks are attached to beryllium substrates, which carry their readout structures, to form wedges. Each disk comprises twelve wedges mounted alternately on the two surfaces of a planar beryllium cooling channel (figure 3 ). Coolant flow cross-section of the channel is $1 \mathrm{~mm} \times 7.5 \mathrm{~mm}$. Readout is at a larger radius than the cooling channel to aid in isolating heat sources from the sensors; the sensor is at a smaller radius. On the $\mathrm{n}$-side of sensors, which faces the cooling channel, a short copper on kapton jumper carries signals from the sensor to the SVX-2 chips. On the p-side, wire bonds connect the sensor to readout chips directly.

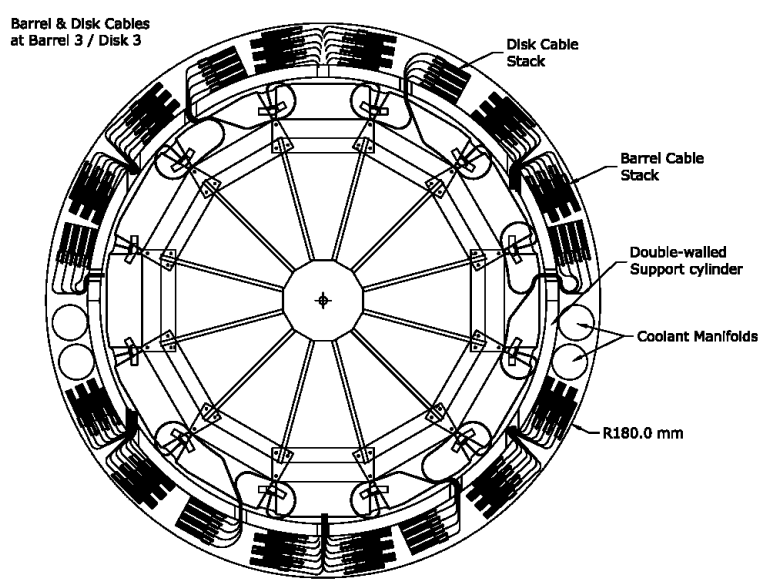

Fig. 3. End view of the associated F-disk. The twelve wedges of the disk, the cooling/support channel, the locations of "outboard" readout HDI's, and pigtail paths are shown. 
Each barrel carries six posts to locate and hold its associated F-disk. Once joined, the barrel and F-disk are handled as a single unit. The three outer F-disks are joined to one another via posts to form an end F-disk module. Adjustable leaf spring mounts support the module from the silicon support cylinder.

H-disks carry twenty-four wedges mounted alternately on the two surfaces of a planar beryllium cooling channel. A relatively complex structure is used to obtain the desired radial coverage and an effective stereo angle of $15^{\circ}$ with single-sided sensors. Two sub-layers, each with an inner radius and an outer radius sensor, are joined back-to-back to form a wedge (figure 4). Back-to-back alignment was accomplished on a specially equipped CMM with a pair of collinear optical probes.

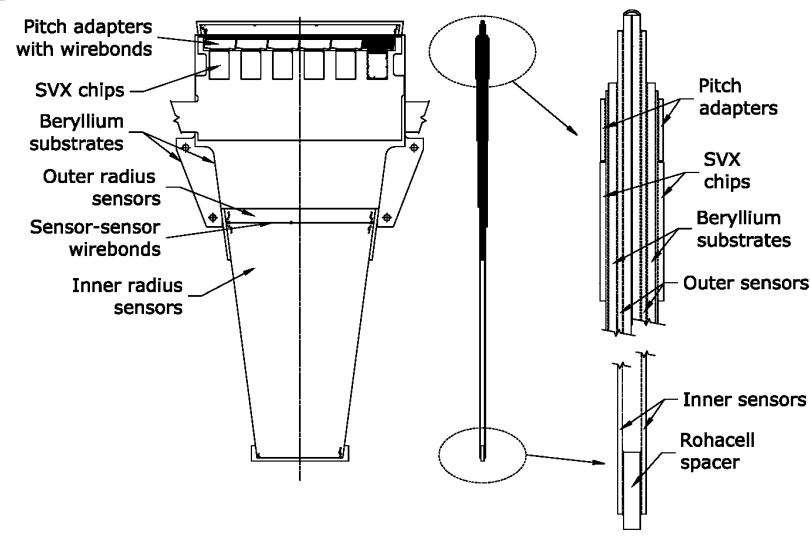

Fig. 4. H-disk wedge. Two half wedge assemblies are joined back-to back to form a wedge. Each half-wedge carries an inner radius and an outer radius sensor. Readout is supported via a beryllium substrate on each outer radius sensor; each substrate overlaps the edge of the inner sensor.

\section{Run IIb silicon}

D0 improvements for Run IIb included the addition of Layer 0 silicon (L0), which has 12288 channels, within the inner radius of Run IIa silicon. A suitable, new beryllium beam pipe had already been obtained in conjunction with an earlier upgrade proposed for Run IIb. Therefore, the transverse space available for L0 was set by Run IIa silicon structures and the new beam pipe.

Openings at six $\mathrm{Z}$ locations in carbon fiber laminate membranes of the Run IIa silicon support structure set the outer limit for L0. Measurements to confirm that the transverse alignment of openings was understood correctly were conducted in fall 2004. Based on those measurements and the need for radial clearance during L0 installation, the maximum radius for $\mathrm{L} 0$ was selected to be $22.02 \mathrm{~mm}$. That provides a minimum radial installation clearance for L0 of $0.09 \mathrm{~mm}$.

Cylindrical portions of the new beam pipe have an outer radius of $14.732 \mathrm{~mm}$ and wall thickness of $0.508 \mathrm{~mm}$. Specially-designed couplings with split flange rings allow a beam pipe flange radius of $15.24 \mathrm{~mm}$. Based upon that, the minimum radius of L0 support structures was selected to be $15.34 \mathrm{~mm}$.

To minimize material near the center of the interaction diamond and fit within the available radial space, readout hybrids were located at each end of a $770 \mathrm{~mm}$ long region populated with sensors. That length ensures that L0 coverage will extend the full length of the Run IIa silicon barrels. A section view at the sensor region is shown in figure 5 .

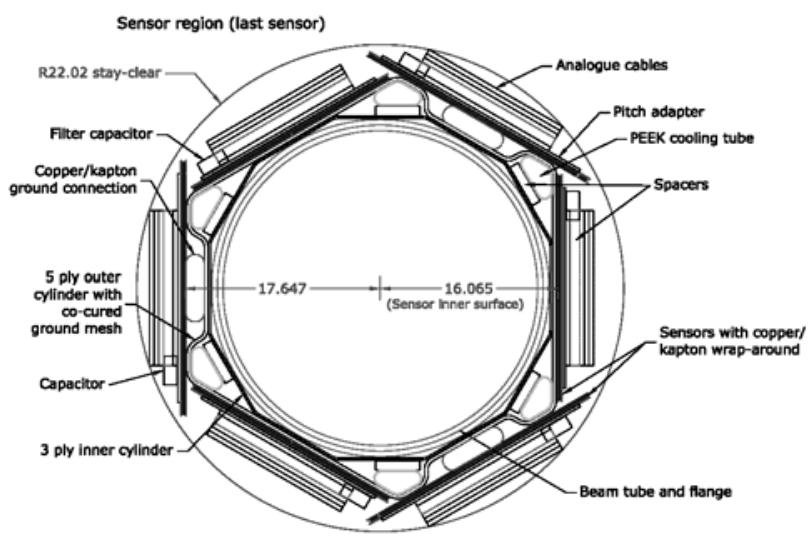

Fig. 5. Section view of the sensor region. Sensor widths and pitches were optimized to match the 256 readout channels provided by a pair of SVX-4 chips while maintaining adequate clearances. Sensor azimuthal overlap is provided by an A- and B- sub-layer geometry.

\subsection{L0 sensors}

All L0 sensors were obtained from Hamamatsu Photonics Corporation (HPK) and have a nominal thickness of $320 \mu \mathrm{m}$. While L0 has only 48 sensors, a larger quantity was obtained to ensure that each of four varieties would be available in sufficient numbers. Sensor electrical quality turned out to be excellent: only three of 120 sensors procured showed small anomalies in $\mathrm{I}-\mathrm{V}$ curves. Sensor flatness and precision of cut edges were also excellent. A-layer sensors have a pitch of 71 $\mu \mathrm{m}$ and a cut width of $20.216 \mathrm{~mm}$; B-layer sensors, a pitch of $81 \mu \mathrm{m}$ and a cut width of $22.776 \mathrm{~mm}$. Of the eight sensors at a given azimuth, the four nearest $Z=0$ have a length of $70 \mathrm{~mm}$; the four further from $Z=0$ have a length of $120 \mathrm{~mm}$.

\subsection{L0 cooling and support structures}

Sensor cooling is provided by flow of an ethylene glycol in water mixture from the existing silicon coolant system through six PEEK tubes, formed between two carbon fiber laminate shells of a support structure. Typical operating temperature of L0 sensors is $-5^{\circ} \mathrm{C}$. One prototype and two final (one to be used and one spare) support structures were fabricated by the University of Washington from Mitsubishi K13C2U carbon fiber impregnated by YLA, Inc. with RS-24 (Mod) epoxy resin. Typical thickness per ply is $0.065 \mathrm{~mm}$. The inner shell extends the full L0 length $(1660 \mathrm{~mm})$ and has a three ply layup of $0^{\circ} / 90^{\circ} / 0^{\circ}\left(0^{\circ}=\right.$ longitudinal $)$. Four sections of outer shells provide mounting surfaces for sensors and their hybrids: 
two sections for sensors either side of $Z=0$ and one for each of the hybrid regions. All outer shells contain five plies with a lay-up of $+20^{\circ} /-20^{\circ} / 0^{\circ} /-20^{\circ} /+20^{\circ}$. Epoxy joins the inner and outer shells, cooling tubes, two PEEK coolant distribution manifolds at each end, and ball and socket mounting pieces together to form the support structure unit.

\subsection{L0 Grounding Provisions}

Grounding structures consisting of copper mesh on $25 \mu \mathrm{m}$ kapton were co-cured with each outer shell. The thickness of copper on each of the two kapton surfaces was $5 \mu \mathrm{m}$; copper coverage was approximately $30 \%$. Copper on kapton jumpers between sensor and hybrid shells ensure a common ground. Copper on kapton circuits which wrap around one end of each sensor provide ground connections from sensors to sensor shell and bias connections to the back surface of each sensor. Two analogue cable layers carry signals from each sensor to its hybrid and carry sensor bias and ground. Kapton mesh spacers ( $75 \mu \mathrm{m}$ thickness, $14 \%$ fill factor) between cables minimize crosstalk. Early design studies on prototypes and design features incorporated to minimize noise and crosstalk are described in [3].

\subsection{LO Modules: Assembly and Installation}

To ensure good transverse alignment of cables, proper longitudinal distance between sensors and hybrids, and alignment of features impacting radial space, sensors and hybrids were combined into modules on a coordinate measuring machine (CMM). A CMM was also used to ensure that sensor/hybrid modules were accurately positioned as they were epoxied to their support structure. A Heidenhain rotary encoder allowed the structure to be rotated so that each module would be at the 12 o'clock position when it was glued into place. CMM measurements were made of the two final support cylinders as a function of load. Corrections were made during module installation to compensate for the expected 27 $\mu \mathrm{m}$ gravitational deflection over the length of the sensor region.

\subsection{L0 Readout}

Readout is based upon SVX-4 chips, which were developed jointly by CDF and D0 for Run IIb. Each sensor is connected via a pair of stacked low-mass analogue cables to a hybrid carrying two SVX-4 chips. Analogue cable lengths ranged from 170 to $320 \mathrm{~mm}$. Pitch adapters match the cable pitch (91 $\mu \mathrm{m})$ to the sensor pitch (71 or $81 \mu \mathrm{m}$, depending on sensor variety) and also provide bias and grounding paths. Low mass digital cables connect the hybrids to "junction cards" in front of the first H-disk. From there, twisted pair cables and clock cables connect to adapter cards on the face of the central calorimeter. The adapter cards match the bipolar control signals needed for SVX-4 chips to the single signals used by SVX-2 chips, thereby allowing the remaining portions of the Run IIa silicon readout chain to be used with SVX-4 chips.
They also provide power isolation, which allowed the use of a L0 carbon fiber support structure with no electrical break at $\mathrm{Z}$ $=0$. At its end mounts the L0 support structure is isolated from Run IIa carbon fiber structures by G-10 connecting rings.

All four H-disks were removed to provide access for L0 installation. To remain within the channel count of the Run IIa readout system, only the two innermost H-disks were reinstalled for Run IIb.

\subsection{Outlook}

L0 has performed nearly flawlessly since it was installed. We look forward to the $8 \mathrm{fb}^{-1}$ integrated luminosity the Tevatron plans to deliver during Run II. With the successful completion, installation, and commissioning of L0, we can do that with confidence that the silicon tracker will perform well for the duration of Run II.

\section{Acknowledgements}

The author wishes to thank D0, Fermilab, the U. S. Department of Energy, and the U. S. National Science foundation for their support, and the workshop organizers for their hospitality and the opportunity to participate

\section{References}

[1] The D0 Upgrade: The Detector and Its Physics, Fermilab PUB-36-357-E (1996).

[2] E. Kajfasz, Nucl. Instr. Meth. A511 (2003) 16.

[3] W. Cooper et al., Nucl. Instr. and Meth. A 550 (2005) 127. 